\title{
Individualized, perioperative, hemodynamic goal-directed therapy in major abdominal surgery (iPEGASUS trial): study protocol for a randomized controlled trial
}

Sandra Funcke', Bernd Saugel ${ }^{1}$, Christian Koch², Dagmar Schulte², Thomas Zajonz², Michael Sander², Angelo Gratarola ${ }^{3}$, Lorenzo Ball3 , Paolo Pelosi ${ }^{3}$, Savino Spadaro ${ }^{4}$, Riccardo Ragazzi ${ }^{4}$, Carlo Alberto Volta ${ }^{4}$, Thomas Mencke ${ }^{5}$, Amelie Zitzmann" ${ }^{5}$, Benedikt Neukirch ${ }^{5}$, Gonzalo Azparren ${ }^{6}$, Marta Giné ${ }^{6}$, Vicky Moral ${ }^{6}$, Hans Otto Pinnschmidt ${ }^{7}$, Oscar Díaz-Cambronero ${ }^{8}$, Maria Jose Alberola Estelles ${ }^{8}$, Marisol Echeverri Velez ${ }^{8}$, Maria Vila Montañes ${ }^{8}$, Javier Belda ${ }^{9}$, Marina Soro ${ }^{9}$, Jaume Puig ${ }^{9}$, Daniel Arnulf Reuter ${ }^{5}$ and Sebastian Alois Haas ${ }^{5^{*}}$ (D)

\begin{abstract}
Background: Postoperative morbidity and mortality in patients undergoing surgery is high, especially in patients who are at risk of complications and undergoing major surgery. We hypothesize that perioperative, algorithmdriven, hemodynamic therapy based on individualized fluid status and cardiac output optimization is able to reduce mortality and postoperative moderate and severe complications as a major determinant of the patients' postoperative quality of life, as well as health care costs.

Methods/design: This is a multi-center, international, prospective, randomized trial in 380 patients undergoing major abdominal surgery including visceral, urological, and gynecological operations. Eligible patients will be randomly allocated to two treatment arms within the participating centers. Patients of the intervention group will be treated perioperatively following a specific hemodynamic therapy algorithm based on pulse-pressure variation (PPV) and individualized optimization of cardiac output assessed by pulse-contour analysis (ProAQT® device; Pulsion Medical Systems, Feldkirchen, Germany). Patients in the control group will be treated according to standard local care based on established basic hemodynamic treatment. The primary endpoint is a composite comprising the occurrence of moderate or severe postoperative complications or death within 28 days post surgery. Secondary endpoints are: (1) the number of moderate and severe postoperative complications in total, per patient and for each individual complication; (2) the occurrence of at least one of these complications on days 1, 3, 5, 7, and 28 in total and for every complication; (3) the days alive and free of mechanical ventilation, vasopressor therapy and renal replacement therapy, length of intensive care unit, and hospital stay at day 7 and day 28; and (4) mortality and quality of life, assessed by the EQ-5D-5 $\mathrm{L}^{\mathrm{TM}}$ questionnaire, after 6 months.
\end{abstract}

Discussion: This is a large, international randomized controlled study evaluating the effect of perioperative, individualized, algorithm-driven ,hemodynamic optimization on postoperative morbidity and mortality.

Trial registration: Trial registration: NCT03021525. Registered on 12 January 2017.

Keywords: Postoperative morbidity, Mortality, Hemodynamic optimization, Individualized medicine, Quality of life

\footnotetext{
* Correspondence: sebastian.haas@med.uni-rostock.de

${ }^{5}$ Department of Anesthesia and Intensive Care Medicine, University of

Rostock, Schillingallee 35, 18057 Rostock, Germany

Full list of author information is available at the end of the article
} 


\section{Background}

In high-risk surgical patients, rates of postoperative complications range from $25 \%$ up to more than $40 \%[1,2]$. These patients not only have reduced functional independence, quality of life and long-term survival [3, 4], moreover, the treatment of postsurgical complications is extremely expensive. In the US the average extra cost for treating a patient developing one or more complication is approximately US\$18,000 [1, 5, 6]. Therefore, reduction of postsurgical complications with fewer days in an intensive care unit, fewer days on organ support, reduction of resource utilization and reduction of hospital stay would reduce costs also for the health care system. Inadequate supply of oxygen is one of the major factors leading to postoperative organ dysfunction and complications. Oxygen supply is mainly determined by cardiac output rather than blood pressure, which, however, has been the primary hemodynamic target during surgery for decades and still is. In this context hemodynamic goal-directed therapy, focusing on an optimization of fluid status and cardiac output, is believed to have the ability to reduce postoperative complications. Since methods like thermodilution, arterial pulsecontour analysis or esophageal Doppler allow routine measurement of cardiac output, several single-center studies have brought up the first evidence that perioperative, algorithm-driven optimization of cardiac output can reduce postoperative complications and improve outcome [7-11]. Also meta-analyses have demonstrated a reduction of morbidity in patients undergoing high-risk surgery [12-17]. However, comparability of those studies is limited due to inhomogeneity in population and therapeutic approaches. Moreover, in most published trials so far, hemodynamic goals had been predefined independently from the individual need of the single patient and further generalized to an entire study population, i.e., either dedicated values of stroke volume or cardiac output, or a standardized maximization of stroke volume by volume loading were used for all patients.

The focus in previous studies was put on implementation of fixed values of hemodynamic parameters, disregarding the single patient's individual cardiovascular capacities, especially the individual range of cardiac output.

The hypothesis of the planned clinical trial is that perioperative hemodynamic optimization based on the novel approach of individualization of hemodynamic therapy can reduce postoperative morbidity and mortality.

\section{Methods/design}

\section{Justification of the study}

The present study aims to evaluate the impact of perioperative, algorithm-driven, hemodynamic therapy based on individualized fluid status and individualized cardiac output optimization on postoperative moderate and severe complications and mortality. The study is justified by the high rate of postoperative complications as a major determinant for the reduction of quality of life for the affected patients and high costs for the health system which may be reduced by individualized, perioperative hemodynamic therapy.

\section{Study design}

This is a prospective, multi-center, international, randomized controlled clinical trial in 380 patients scheduled for major abdominal surgery in six European centers having a large number of patients eligible for study inclusion (see Appendix). The study coordination will be conducted by a team of principle investigators (PI), Daniel A. Reuter, Sebastian A. Haas, Sandra Funcke, and Bernd Saugel, from the University Medical Center Hamburg-Eppendorf, Germany and from the Rostock University Medical Center, Germany.

The trial was designed in accordance with the fundamental principles established in the Declaration of Helsinki and within the requirements established by the German legislation in the field of biomedical research, the protection of personal data, and bioethics. The study was registered in January 2017 (received on 12 January 2017) at https://clinicaltrials.gov with the identification number NCT03021525. The study was approved by the Ethics Committee Giessen, Germany in February 2017 acting as the overall Ethical Review Board for this study (Additional file 1). Further, we can specify that all participating centers will obtain the approval from the local Ethical Review Board according to individual legislation regulations. Before inclusion into the study, written informed consent from the patient will be obtained (Additional file 2). See Additional file 3 for the Standard Protocol Items: Recommendations for Interventional Trials (SPIRIT) Checklist of the study protocol and Fig 1. for the adapted SPIRIT Figure.

After an initial meeting of the principle investigators (PIs) together with all local main investigators (LMI) the centers are visited at the partner hospital and trained with regard to the process of randomization, enrollment, data acquisition, and treatment strategies.

\section{Study population}

To be eligible for inclusion into this study (day 0), each patient must fulfill all the inclusion criteria during screening and prior to enrollment into the trial.

\section{Inclusion criteria}

To ensure the presence of marked surgical trauma, open visceral, urological, and gynecological surgery are covered by this study. Expected duration of surgery must be $\geq 120 \mathrm{~min}$ and the requirement of volume therapy must be expected to be $\geq 21$. Risk for any postoperative complications must be $\geq 10 \%$ as assessed preoperatively by 


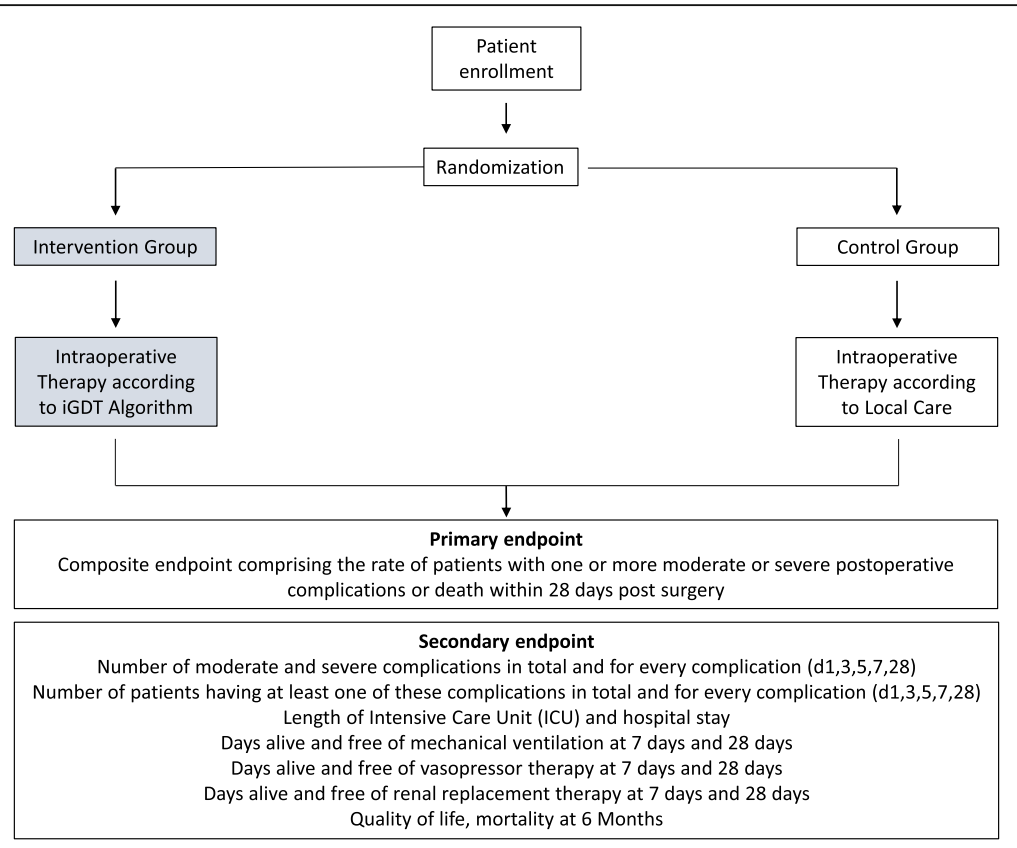

Fig. 1 Study flow chart. iGDT: individualized, goal-directed therapy

the ACS-NSQIP (American College of Surgery - National Surgical Quality Improvement Program, www. acsnsqip.org) risk calculator $[4,18,19]$.

\section{Exclusion criteria}

Patients aged $<18$ years, use of a laparoscopic approach, patients who are not in sinus rhythm, patients having highly impaired left ventricular function (ejection fraction $<30 \%$ ) or severe aortic valve stenosis (aortic valve area $<1 \mathrm{~cm}^{2}$, mean gradient $>40 \mathrm{mmHg}$ ), pregnant women, emergency surgeries (surgery required within $24 \mathrm{~h}$ ), primarily vascular surgery, patients suffering from septic shock, patients having pheochromocytoma, patients suffering from non-cardiac chest pain, patients suffering from anuric renal failure, those refusing to consent, patients receiving palliative treatment only (likely to die within 6 months) and patients suffering from acute myocardial ischemia (within 30 days before randomization) are excluded from the study. Further, in case that clinicians intended to use cardiac output monitoring for clinical reasons patients should also not be included in the study.

\section{Randomization}

The use of an electronic Case Report Form (eCRF) including randomization software ensures a safe and prompt randomization via computer. Participants will be centrally allocated to treatment groups (allocation ratio: $1: 1$ ) by the eCRF-generated randomization procedure (randomization in blocks of 6). This ensures a balanced allocation with respect to centers and treatment arms. The study is summarized in Fig. 1.

\section{General care and procedures for the control and intervention groups}

\section{General information on patients' treatment}

Treatment besides hemodynamic optimization in both the control and the intervention group is defined based on the respective guidelines and recommendations of the medical societies to avoid extremes of clinical practice. Oxygenation will be targeted for a $\mathrm{SpO}_{2}$ of $94 \%$ or higher by choosing an appropriate level of inspired oxygen. The tidal volume will be set at $6-8 \mathrm{ml} / \mathrm{kg}$ and PEEP at $0-10 \mathrm{~cm}$ $\mathrm{H}_{2} \mathrm{O}$ according to clinical needs and the decision of the physician in charge. The target for heart rate is below $100 \mathrm{bpm}$, the target range for mean arterial blood pressure is $65 \mathrm{mmHg}$ or higher, using vasopressors as required. Core temperature will be maintained at $>36 \mathrm{C}$ by continuous heat application to the patient and fluid warming. Choice of fluid is determined on the basis of the recent guidelines [20] for intravascular volume therapy. Arterial or venous blood samples are taken regularly for assessment of hemoglobin, oxygenation, decarboxylation, electrolytes, and lactate. An epidural catheter is placed before induction of anesthesia at the discretion of the treating anesthesiologist. Perioperative analgesia will be provided by epidural or intravenous infusion of analgesics according to the standards of the participating centers. Blood products will be transfused according to the Guidelines from the European Society of Anesthesiology (for an overview see Additional file 4: Table S1) [21]. 


\section{Treatment of the control group}

Patients in the control group will be treated according to established basic treatment goals as described in the general information on patient treatment (heart rate $<100 \mathrm{bpm}$, mean arterial pressure $>65 \mathrm{mmHg}, \mathrm{SpO}_{2}>94 \%$, and core temperature $>36 \mathrm{C}$ ). In the control group, no specific vasopressor is prescribed to achieve the mean arterial pressure goal and the choice of drug is at the discretion of the treating anesthesiologist. Basic anesthesiological monitoring by five-lead electrocardiogram, pulse oximetry, non-invasive blood pressure monitoring and capnography is performed in every patient. Placement of an arterial and central venous line is at the discretion of the treating clinician, as is the decision to administer fluids and catecholamines. After extubation, hemodynamic treatment in the recovery room is at the discretion of the treating anesthesiologist either by administration of fluids, catecholamines, or other drugs as required.

\section{Treatment of the intervention group}

The trial intervention period will commence from the induction of anesthesia until $8 \mathrm{~h}$ after surgery, or until discharge from the postoperative intensive or intermediate care unit. Drugs of choice are dobutamine for inotropic support and norepinephrine as a vasopressor. No other catecholamines should be used in the intervention group. Hemodynamic management is performed according to standard treatment until an arterial catheter is placed. The signal from the arterial catheter is then processed by noncalibrated pulse-contour analysis (ProAQT@; Pulsion Medical Systems, Feldkirchen, Germany) for pulsepressure variation (PPV) and cardica index $(\mathrm{CI})$ measurement. A fluid challenge will be performed by infusion of $500 \mathrm{ml}$ in $<15 \mathrm{~min}$. The choice of fluid in case of indicated fluid loading is determined on the basis of the recent guidelines for volume therapy [20].

\section{Initial assessment of individually optimized $\mathrm{Cl}$}

When arterial pulse-contour analysis is implemented, patients receive an initial hemodynamic assessment based on PPV and CI in order to identify individual values of optimal CI as shown in algorithm 1 (Fig. 2).

First, fluid status is optimized by fluid loading $(500 \mathrm{ml}$ in $<15 \mathrm{~min}$ ) until PPV is $<12 \%$. At least $15 \mathrm{~min}$ after induction of general anesthesia, after individual optimization of the fluid status, the patient's individual optimal CI in the specific situation of general anesthesia is defined and used as the individual hemodynamic goal until the end of the intervention period. Only if this value is below $2.5 \mathrm{l} / \mathrm{min} / \mathrm{m}^{2}$ inotropes are administered to reach this minimum $\mathrm{CI}$ to prevent patients from developing a low cardiac output.

\section{Hemodynamic assessment during mechanical ventilation}

After the initial determination of the individual optimal CI, further hemodynamic assessment during mechanical ventilation and surgery is performed every $15 \mathrm{~min}$, or if mechanical ventilation is continued after surgery every $30 \mathrm{~min}$, or if required due to hemodynamic instability at any time according to algorithm 2 (Fig. 3). Hemodynamic parameters are documented every $30 \mathrm{~min}$ during surgery and every 60 min postoperatively.

If the $\mathrm{CI}$ is below the initially assessed individual optimal $\mathrm{CI}$, again fluid status is evaluated based on PPV. Fluids are administered as long as PPV is higher than $12 \%$ and the CI increases. Again, inotropes are used when fluid optimization does not result in realization of the individual optimal CI. This treatment algorithm is always reassessed when the $\mathrm{CI}$ drops below the individually optimized CI.

\section{Hemodynamic assessment after extubation (maximum $8 h$ after surgery)}

Because of spontaneous breathing, after extubation PPV is not usable anymore for fluid status evaluation. Hemodynamic assessment is then performed according to algorithm 3 (Fig. 4). Hemodynamic assessment after extubation is repeated every $30 \mathrm{~min}$, or if required due to hemodynamic instability at any time according to algorithm 3. Hemodynamic parameters are documented every $60 \mathrm{~min}$.

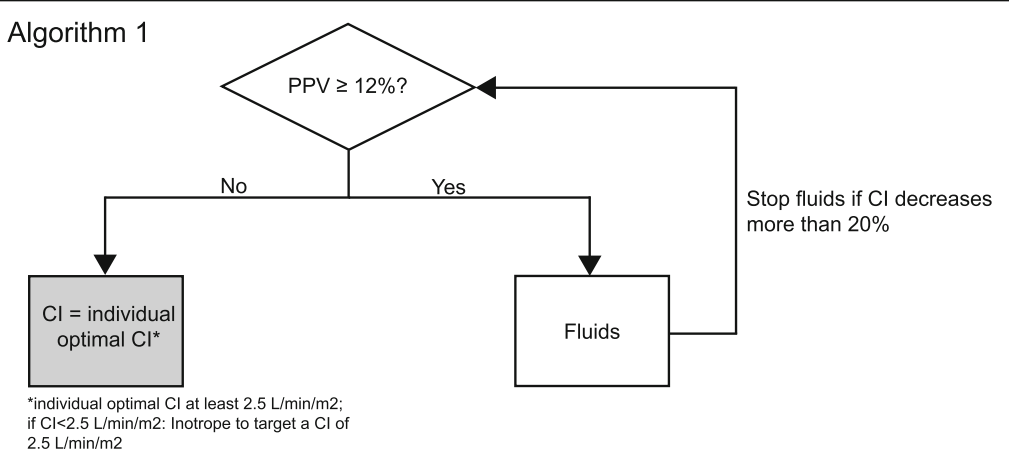

Fig. 2 Algorithm 1. Intervention algorithm of initial assessment of individually optimized Cardiac Index (CI). Cl: Cardiac index; PPV: Pulse pressure variation 


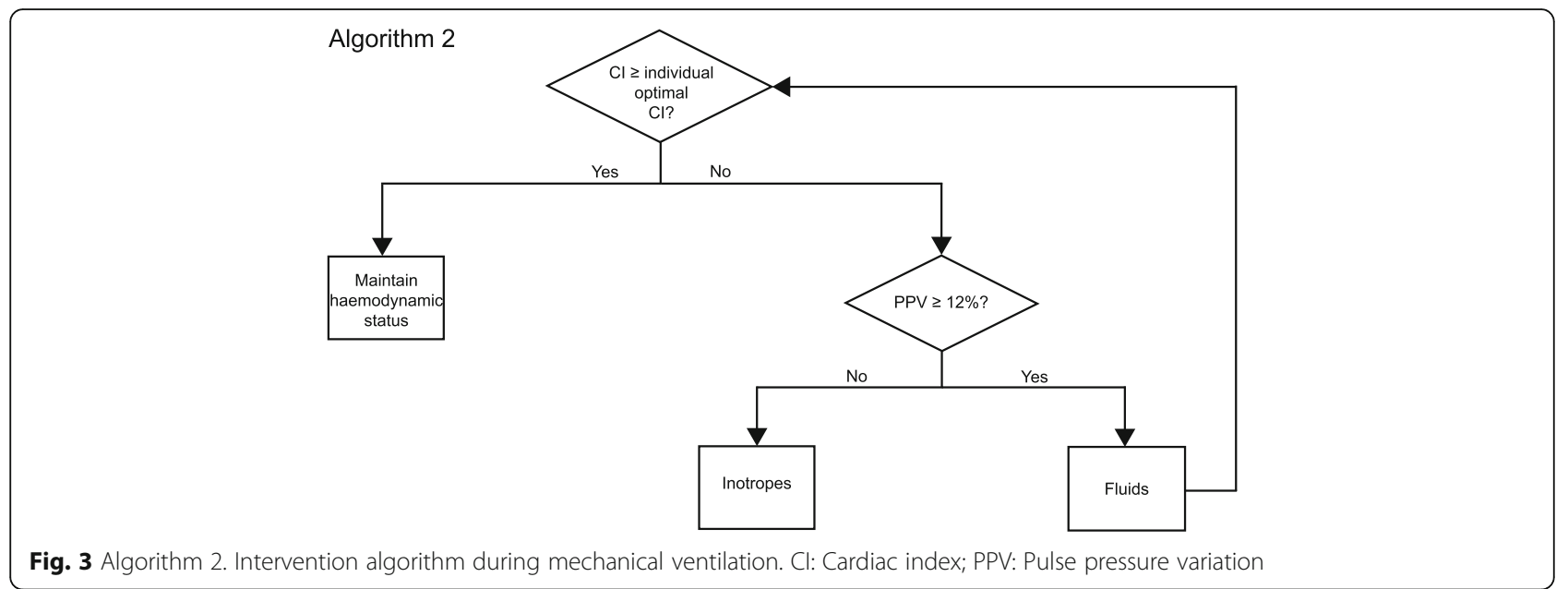

When the $\mathrm{CI}$ is below the individually determined optimal CI, a fluid challenge ( $500 \mathrm{ml}$ in $<15 \mathrm{~min}$ ) is performed. If the increase of $\mathrm{CI}$ is higher than $15 \%$, a fluid challenge of $500 \mathrm{ml}$ is repeated. If the increase of the CI is below $15 \%$, or the $\mathrm{CI}$ even decreases, inotropes should be started or increased.

\section{Primary and secondary outcomes Primary endpoint}

Considering the high relevance for the individual patient and for society, the postoperative outcome is most appropriately reflected by a combination of morbidity and mortality. Therefore, the primary endpoint is a composite comprising the rate of patients with one or more moderate or severe postoperative complications or death within 28 days post surgery. Moderate and severe postoperative complications are defined by 22 singleorgan outcome failures based on a consensus statement of the European Society of Anesthesiology (ESA) - European Society of Intensive Care Medicine (ESICM) joint taskforce on perioperative outcome measures [22]. A summary of the included organ failures and the severity grading is given in Additional file 5: Table S2.

\section{Secondary endpoints}

Secondary endpoints are: (1) the number of moderate and severe postoperative complications in total, per patient and for each individual complication; (2) the

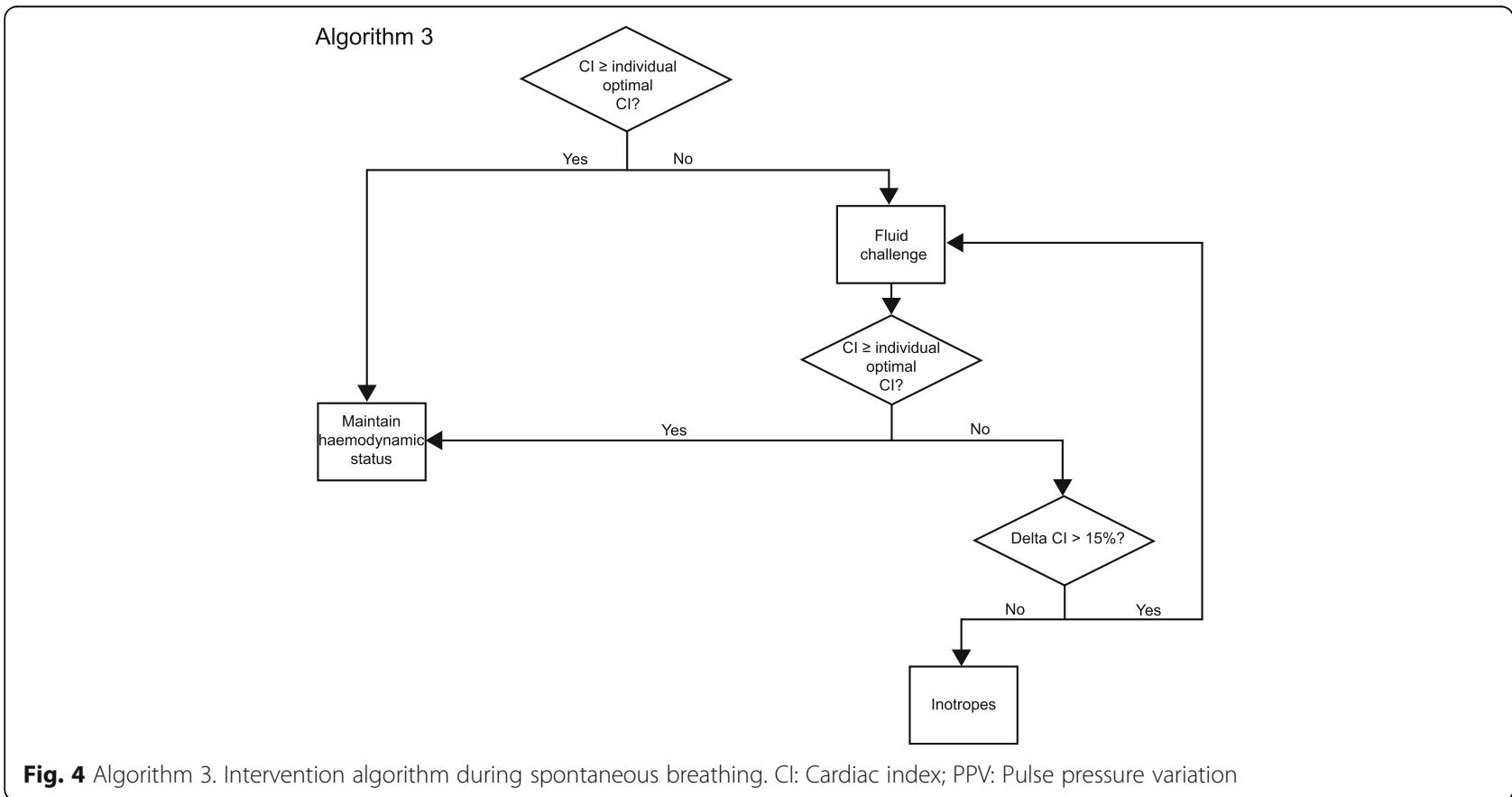


occurrence of at least one of these complications on days $1,3,5,7$, and 28 in total and for every complication. The additional secondary endpoints include (3) days alive and free of mechanical ventilation, vasopressor therapy and renal replacement therapy, length of ICU and hospital stay at day 7 and day 28 further characterize perioperative morbidity and its socioeconomic impacts. (4) For the assessment of quality of life, an interview (personal or by telephone) based on the EQ-5D-5L ${ }^{\mathrm{mm}}$ questionnaire [23] is performed at the time point of enrollment and 6 months after surgery. Mortality is assessed 6 months after surgery. All the secondary endpoints mentioned above will be recorded at the defined time points, but will be analyzed at the end of the study.

\section{Intervention scheme}

The intervention schemes are given in Figs. 2, 3, and 4. The schedule of events is summarized in Fig. 5.

\section{Documentation and data acquisition}

Data will be obtained by a web-based eCRF. Study centers will be trained on the use of the eCRF before initiating a patient's enrollment. To enter a patient into the iPEGASUS trial, research staff at the site will $\log$ on to a secure, web-based randomization system via a link to the respective website and complete the patient's details to obtain a unique patient number and allocation to a treatment group. The local main investigator (LMI) will then be informed about the patient's enrollment. For correct patient data follow-up only the LMI will set up a list with the possibility to retrace the patient's history. Personalized data will be handled by the LMI according to Good Clinical Practice (GCP) requirements. In contrast, data in the eCRF, visible only for the coordinating investigators, will be completely anonymized and not be re-traceable. Data management of the eCRF will be performed by a company specialized in full service study data management (MedSurv GmbH, Nidderau, Germany).

To reduce bias resulting from lack of experience in the use of the protocol, the first two enrolled patients of each center (one allocated to the control group, one allocated to the intervention group) will be withdrawn from the study. Source data verification, quality assurance and monitoring of data during on-site monitoring visits will be performed by the dedicated monitoring team with appropriate frequency according to center performance. The monitoring team will be provided by the Hamburg-Eppendorf University Medical Center.

\section{Methods against bias}

Due to the nature of the intervention, blinding to all types of staff involved in the study is not possible. Primary and secondary outcome parameters will be assessed by a local investigator, on days $1,3,5,7$, and on day 28 , blinded to allocation. Moderate and severe complications will additionally be verified by the LMI or a designee. Source data verification will be performed by

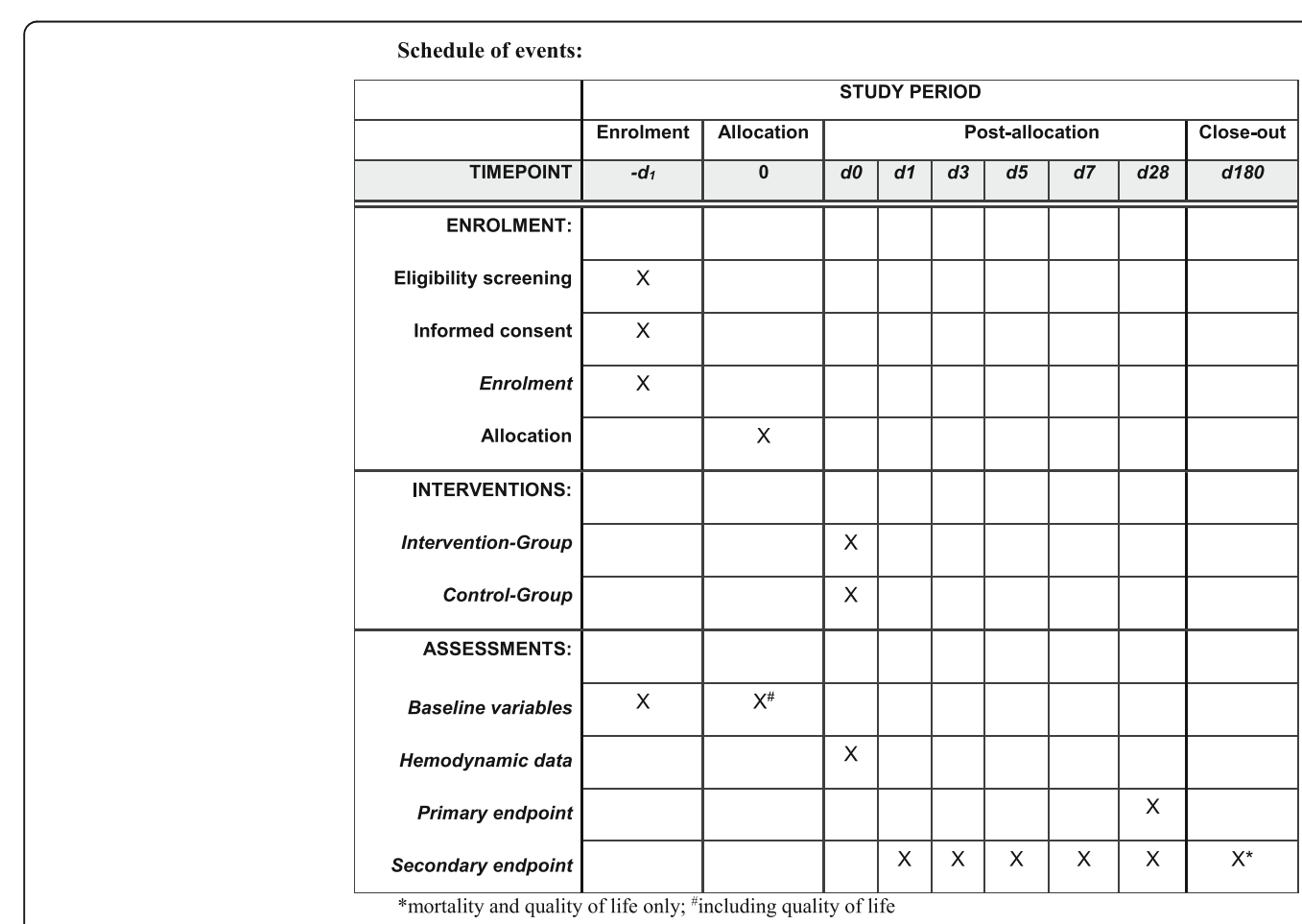

Fig. 5 Schedule of events 
the monitors by regular on-site monitoring visits. To minimize bias provoked by learning principles of goaldirected therapy in the past, and thereby influencing control group treatment, predominately hospitals without prior regular use of cardiac output monitoring in the respective procedures were selected. Further, the inclusion of non-university centers will allow better comparison of the study group to the broad clinical reality.

\section{Compliance/rate of loss to follow-up}

After enrollment of the patient's address, telephone number, and contact information of the primary care physician, a relative of the patient will also be registered enabling us to maintain contact. If patients do not reach the goals of the inclusion criteria (time of surgery $>$ 120 min or volume therapy $>2$ l) they will be excluded from the study subsequently. Visits by a local investigator will be performed on days $1,3,5,7$, and 28 to assess postoperative complications necessary for primary and secondary endpoints. However, if patients are discharged from hospital to home, to a secondary hospital, or to a rehabilitation facility before day 28 , final determination of all parameters relevant for the primary and the secondary endpoints will be assessed on day 28 by a structured telephone interview with the secondary hospital, the patient or the rehabilitation facility or the primary care physician. Due to the lack of subsequent examinations of the patient after day 28 , loss to follow-up is unlikely. However, to reflect an unscheduled loss to followup an overall dropout rate of $10 \%$ is already included in the sample size calculation.

\section{Sample size calculation and statistical analysis Sample size}

According to previous published data [24] assessing the impact of hemodynamic goal-directed therapy, we assume that the primary outcome will occur in $48 \%$ of the control group and in 33\% of the intervention group. The null hypothesis of the study is that individualized, goaldirected therapy does not lead to a statistically significant difference in morbidity and mortality at day 28 after major abdominal surgery. Assuming in this null hypothesis (H0) that the two groups are not significantly different from each other at the significance level $\alpha=0.05$ with respect to the primary outcome, 334 patients are required to have an $80 \%$ chance of detecting a difference between the control group and the experimental group of 15\%. Therefore, 167 patients per group would have to be enrolled into the study. Taking into account a dropout rate of about $10 \%$ and exclusion of the first two patients in every center, a number of $n=380$ patients is necessary.

\section{Primary and secondary endpoints}

Baseline sample characteristics will be shown for all randomized patients treatment-wise and for pooled data. For categorical variables, category frequency counts and percentages will be shown. For continuous variables, the arithmetic mean, median, 1st and 3rd quartiles, minimum and maximum values will be presented. Follow-up data will be given in line plots allowing visual comparisons between treatment groups.

\section{Primary analysis}

For the primary endpoint "rate of moderate or severe postoperative complication or death within 28 days post surgery," the fixed effect of treatment and the random effect of clinic and the treatment slope will be estimated by a binary logistic regression model. The resulting odds ratio with corresponding 95\% confidence intervals (CI) and $p$ values will be tabulated. Estimated marginal frequencies and their 95\% CIs will be presented graphically. Superiority of the individualized hemodynamic therapy is considered established if the upper margin of the 95\% $\mathrm{CI}$ of the corresponding odds ratio lies below 1 .

\section{Secondary analyses}

The composite endpoint "occurrence of moderate or severe postoperative complication or death" and the secondary endpoints "postoperative morbidity," "occurrence of moderate or severe complications," "occurrence of individual complications" and "number of complications per patient" will be analyzed via longitudinal binary logistic regression models, treating clinic, treatment slope and patient within clinic as random effects. Treatment, frequencies over time (FU) and their interaction will be considered fixed effects. Odds ratios, their 95\% CIs and $p$ values will be tabulated. Estimated marginal $\mathrm{FU}$ and their 95\% CIs will be presented graphically.

\section{Discussion}

Reduction of perioperative mortality and morbidity is greatly required due to the massive benefit for the patients and the health care system. In Europe, overall mortality after surgery has been reported to be up to $4 \%$ [2] and after high-risk surgery or in high-risk patients it has to be assumed to be even higher. Morbidity or postoperative complications, which are potentially triggered by inadequate oxygen supply to the peripheral organs, have to be seen as the primary cause for reduction of quality of life and the immense health care costs ensuing. Every percentage point reduction of postoperative complications could save approximately 180 million to US\$270 million [5]. In a recent multi-center trial the achievement of adequate preoperative oxygen delivery was associated with a lower postoperative morbidity compared to the standard of care [25]. In this context a 
various number of meta-analyses evaluating the impact of hemodynamic goal-directed therapy, and thereby optimizing oxygen supply, have already demonstrated reduction of morbidity in patients undergoing high-risk surgery [12-17]. However, meta-analyses are frequently limited in their statement due to inhomogeneity in population and therapeutic approaches. In published trials, stroke volume or cardiac output have been predefined, standardized and generalized to a total cohort of patients not taking into account the single patient's individual cardiovascular needs and capacities. In the largest randomized multi-center study on goal-directed therapy published so far, the focus of the intervention protocol was maximization of stroke volume by repetitive fluid administration without using functional parameters of preload supplemented by a fixed dose of inotropes. In this trial the focus was put on maximizing stroke volume. However, this large multi-center trial did not significantly reduce the composite primary endpoint of morbidity and mortality [26]. In contrast to this approach, in the iPEGASUS trial, the protocol is not targeting a maximized stroke volume. In the iPEGASUS trial, individual CI assessment is primarily based on PPVguided volume status optimization followed by defining the achieved $\mathrm{CI}$ as "individually optimized $\mathrm{CI}^{\text {" under opti- }}$ mized volume status conditions. This "individually optimized CI" is used as the CI target during the total perioperative period and volume and inotrope application is only performed when this "individually optimized CI" is not reached.

The hypothesis of the planned clinical trial is that perioperative hemodynamic optimization, based on the novel approach of individualization of hemodynamic therapy, can reduce postoperative morbidity and also mortality. A previous study focusing on a PPV-guided CI individualization and, in contrast to iPEGASUS, using PPV-based volume loading, even when the individualized $\mathrm{CI}$ was reached, demonstrated a reduction in postoperative complications [24]. However, this finding is now evaluated in a larger randomized controlled, multi-center study using a modified intervention protocol. Thus, in the proposed study, hemodynamic therapy is tailored individually to each patient, based on optimization of fluid status by the functional parameter PPV and based on this individually titrated goal of CI. Individualization of therapy is a key factor for successful treatment, especially when reduction of morbidity and mortality is targeted. Therefore, the iPEGASUS trial further develops the concept of hemodynamic goal-directed therapy to individually set goals and is designed to assess its impact on mortality and morbidity.

\section{Trial status}

Recruitment started in August 2017 and is anticipated to be completed by the end of 2018 .

\section{Appendix}

These are the collaborators in the European centers initiated for patient recruitment and enrollment:

1. Policlinico San Martino Hospital, IRCCS for Oncology, University of Genoa, Genoa, Italy

2. Department of Morphology, Surgery and Experimental Medicine, Intensive Care Unit University of Ferrara, Sant'Anna Hospital, Via Aldo Moro, 8, 44,121, Ferrara, Italy

3. Department of Anesthesiology, Intensive Care Medicine and Pain Therapy, Universitätsklinikum Giessen und Marburg GmbH, Giessen, Germany

4. Department of Anesthesia and Intensive Care Medicine, University of Rostock, Schillingallee 35, Rostock 18,057, Germany

5. Department of Anesthesiology, Hospital Universitari i Politécnic La Fe, Valencia, Spain

6. Department of Anesthesiology, Hospital Santa Creu i Sant Pau, C/ Mas Casanovas 90, 08041, Barcelona, Spain

\section{Additional files}

Additional file 1: Ethical approval. (PDF $386 \mathrm{~kb}$ )

Additional file 2: Written informed consent. (PDF 53 kb)

Additional file 3: SPIRIT 2013 Checklist. (DOC $121 \mathrm{~kb}$ )

Additional file 4: Table S1. Management of severe bleeding - Extract from the Guidelines from the European Society of Anaesthesiology. (PDF $258 \mathrm{~kb}$ )

Additional file 5: Table S2. Overview of Single Organ Outcome Failures and Severity Grading. (PDF $182 \mathrm{~kb}$ )

\section{Abbreviations}

Cl: Cardiac Index; eCRF: Electronic Case Report Form; ERB: Ethical Review Board; ESA: European Society of Anesthesiology; ESICM: European Society of Intensive Care Medicine; ICU: Intensive care unit; iGDT: Individualized, goaldirected therapy; LMI: Local main investigator; MAP: Mean arterial pressure; PI: principle investigators; PPV: Pulse-pressure variation

\section{Acknowledgements}

We thank Frank Ponndorf (MedSurv GmbH, Nidderau, Germany) for setup and continuous care of the eCRF. Further, we thank EuroQol for providing us with the quality of life questionnaire.

\section{Funding}

This trial is supported by an unrestricted research grant by the Getinge Group, Pulsion Medical Systems SE, Hans-Riedl Str. 2, 85622 Feldkirchen, Germany. The funders have no role in the content, decision to publish, or preparation of the manuscript. None of the clinical investigators enrolling patients have received any honoraria for participating in the study.

\section{Availability of data and materials}

All data being generated and analyzed during this study can be obtained from the eCRF dataset system provided by the MedSurv GmbH, Nidderau, Germany or from the corresponding author on reasonable request.

\section{Authors' contributions}

SF: development of the study protocol, study design, obtaining ethical approval, eCRF setup, manuscript writing, statistical design. BS: development of the study protocol, study design, manuscript writing. CK: development of the study protocol, study design, manuscript writing. DS: development of 
the study protocol, study design, manuscript writing. TZ: development of the study protocol, study design, manuscript writing. MS: development of the study protocol, study design, manuscript writing, obtaining ethical approval. AG: development of the study protocol, study design, manuscript writing. LB: development of the study protocol, study design, manuscript writing, obtaining ethical approval. PP: development of the study protocol, study design, manuscript writing. SS: development of the study protocol, study design, manuscript writing. RR: development of the study protocol, study design, manuscript writing. CAV: development of the study protocol, study design, manuscript writing, obtaining ethical approval. TM: development of the study protocol, study design, manuscript writing, obtaining ethical approval. AZ: development of the study protocol, study design, manuscript writing. BN: development of the study protocol, study design, manuscript writing. GA: development of the study protocol, study design, manuscript writing. MG: development of the study protocol, study design, manuscript writing. VM: development of the study protocol, study design, manuscript writing, obtaining ethical approval. HP: development of the study protocol, study design, manuscript writing, statistical design. ODC development of the study protocol, study design, manuscript writing. MJAE development of the study protocol, study design, manuscript writing. MEV development of the study protocol, study design, manuscript writing. MVM development of the study protocol, study design, manuscript writing. JB: development of the study protocol, study design, manuscript writing, obtaining ethical approval. MS: development of the study protocol, study design, manuscript writing. JP: development of the study protocol, study design, manuscript writing. DAR: development of the study protocol, study design, obtaining ethical approval, eCRF setup, manuscript writing, statistical design. SAH: development of the study protocol, study design, obtaining ethical approval, eCRF setup, manuscript writing, statistical design. All authors read and approved the final manuscript.

\section{Ethics approval and consent to participate}

The Ethical Review Board of the University of Gießen, Germany as overall Ethical Review Board (committee's reference number 257/16) approved the study. Further, all local Ethical Review Boards of all participating centers will also approve the study before enrolling patients. Informed consent will/have be(en) obtained from all participants of the study.

\section{Competing interests}

SF has no competing interests. BS collaborates with Pulsion Medical Systems SE (Feldkirchen, Germany) as a member of the Medical Advisory Board and received honoraria for giving lectures and refunds of travel expenses from Pulsion Medical Systems SE (Feldkirchen, Germany), received institutional research grants, unrestricted research grants, and refunds of travel expenses from Tensys Medical Inc. (San Diego, CA, USA), received honoraria for giving lectures and refunds of travel expenses from CNSystems Medizintechnik AG (Graz, Austria), received research support from Edwards Lifesciences (Irvine, CA, USA). CK has no competing interests. DS has no competing interests. TZ has no competing interests. MS received grants from Masimo, Ratiopharm, Edwards Lifesciences, Getinge Group, AMOMED and Medtronic. DAR is member of the Medical Advisory Board of Pulsion SE. AG, LB, PP, SS, RR, CAV, TM, AZ, BN, GA, MG, VM, HOP, ODC, MJAE, MEV, MVM, JB, MS, JP, and SAH have no competing interests.

\section{Publisher's Note}

Springer Nature remains neutral with regard to jurisdictional claims in published maps and institutional affiliations.

\footnotetext{
Author details

'Department of Anesthesiology, Center of Anesthesiology and Intensive Care Medicine, University Medical Center Hamburg-Eppendorf, 20246 Hamburg, Germany. ${ }^{2}$ Department of Anesthesiology, Intensive Care Medicine and Pain Therapy, Universitätsklinikum Giessen und Marburg GmbH, 35392 Giessen, Germany. ${ }^{3}$ Department of Surgical Sciences and Integrated Diagnostics, San Martino Policlinico Hospital, IRCCS for Oncology, University of Genoa, Genoa, Italy. ${ }^{4}$ Department of Anesthesia and Intensive Care, University of Ferrara, Sant Anna Hospital, Via Aldo Moro, 8, 44121 Ferrara, Italy. ${ }^{5}$ Department of Anesthesia and Intensive Care Medicine, University of Rostock, Schillingallee 35, 18057 Rostock, Germany. ${ }^{6}$ Department of Anesthesiology, Hospital Santa Creu i Sant Pau, C/ Mas Casanovas 90, 08041 Barcelona, Spain. ${ }^{7}$ Institute of Medical Biometry and Epidemiology, University Medical Center
}

Hamburg-Eppendorf, 20246 Hamburg, Germany. ${ }^{8}$ Department of Anaesthesiology, Perioperative Medicine Research Group, Hospital Universitari i Politecnic La Fe, Instituto de Investigación Sanitaria La Fe (IIS laFe), Valencia, Spain. ${ }^{9}$ Department of Anesthesiology, Hospital Clínico Universitario de Valencia, Avda. Blasco Ibañez 17, 46010 Valencia, Spain.

Received: 26 October 2017 Accepted: 28 March 2018

Published online: 09 May 2018

\section{References}

1. Ghaferi AA, Birkmeyer JD, Dimick JB. Variation in hospital mortality associated with inpatient surgery. N Engl J Med. 2009;361:1368-75.

2. Pearse RM, Moreno RP, Bauer P, Pelosi P, Metnitz P, Spies C, et al. Mortality after surgery in Europe: a 7 day cohort study. Lancet. 2012;380:1059-65.

3. Jencks SF, Williams MV, Coleman EA. Rehospitalizations among patients in the Medicare fee-for-service program. N Engl J Med. 2009;360:1418-28.

4. Khuri SF, Henderson WG, DePalma RG, Mosca C, Healey NA, Kumbhani DJ, et al. Determinants of long-term survival after major surgery and the adverse effect of postoperative complications. Ann Surg. 2005;242:326-41. discussion 41-3

5. Ebm C, Cecconi M, Sutton L, Rhodes A. A cost-effectiveness analysis of postoperative goal-directed therapy for high-risk surgical patients. Crit Care Med. 2014;42:1194-203.

6. Manecke GR, Asemota A, Michard F. Tackling the economic burden of postsurgical complications: would perioperative goal-directed fluid therapy help? Crit Care. 2014;18:566.

7. Shoemaker WC, Kram HB, Appel PL, Fleming AW. The efficacy of central venous and pulmonary artery catheters and therapy based upon them in reducing mortality and morbidity. Arch Surg. 1990;125:1332-7. discussion 7-8

8. Benes J, Giglio M, Brienza N, Michard F. The effects of goal directed fluid therapy based on dynamic parameters on post-surgical outcome: a metaanalysis of randomized controlled trials. Crit Care. 2014;18:584.

9. Goepfert MS, Richter HP, Zu Eulenburg C, Gruetzmacher J, Rafflenbeul E, Roeher K, et al. Individually optimized hemodynamic therapy reduces complications and length of stay in the intensive care unit: a prospective, randomized controlled trial. Anesthesiology. 2013;119:824-36.

10. Wakeling HG, McFall MR, Jenkins CS, Woods WG, Miles WF, Barclay $G R$, et al. Intraoperative oesophageal Doppler guided fluid management shortens postoperative hospital stay after major bowel surgery. Br J Anaesth. 2005;95:634-42.

11. Kaufmann KB, Stein L, Bogatyreva L, Ulbrich F, Kaifi JT, Hauschke D, et al. Oesophageal Doppler quided goal-directed haemodynamic therapy in thoracic surgery - a single centre randomized parallel-arm trial. $\mathrm{Br} J$ Anaesth. 2017;118:852-61.

12. Aya HD, Cecconi M, Hamilton M, Rhodes A. Goal-directed therapy in cardiac surgery: a systematic review and meta-analysis. Br J Anaesth. 2013;1 10:510-7.

13. Benes J, Zatloukal J, Simanova A, Chytra I, Kasal E. Cost analysis of the stroke volume variation guided perioperative hemodynamic optimization -an economic evaluation of the SWOPT trial results. BMC Anesthesiol. 2014;14:40.

14. Grocott MP, Dushianthan A, Hamilton MA, Mythen MG, Harrison D, Rowan $\mathrm{K}$, et al. Perioperative increase in global blood flow to explicit defined goals and outcomes after surgery: a Cochrane Systematic Review. Br J Anaesth. 2013:111:535-48.

15. Gurgel ST, do Nascimento P Jr. Maintaining tissue perfusion in high-risk surgical patients: a systematic review of randomized clinical trials. Anesth Analg. 2011;112:1384-91.

16. Hamilton MA, Cecconi M, Rhodes A. A systematic review and meta-analysis on the use of preemptive hemodynamic intervention to improve postoperative outcomes in moderate and high-risk surgical patients. Anesth Analg. 2011;112:1392-402.

17. Sun Y, Chai F, Pan C, Romeiser JL, Gan TJ. Effect of perioperative goaldirected hemodynamic therapy on postoperative recovery following major abdominal surgery-a systematic review and meta-analysis of randomized controlled trials. Crit Care. 2017;21:141.

18. Khuri SF, Daley J, Henderson W, Hur K, Demakis J, Aust JB, et al. The Department of Veterans Affairs' NSQIP: the first national, validated, outcome-based, risk-adjusted, and peer-controlled program for the measurement and enhancement of the quality of surgical care. National VA Surgical Quality Improvement Program. Ann Surg. 1998;228:491-507. 
19. Khuri SF, Daley J, Henderson W, Hur K, Gibbs JO, Barbour G, et al. Risk adjustment of the postoperative mortality rate for the comparative assessment of the quality of surgical care: results of the National Veterans Affairs Surgical Risk Study. J Am Coll Surg. 1997;185:315-27.

20. Marx G, Schindler AW, Mosch C, Albers J, Bauer M, Gnass I, et al. Intravascular volume therapy in adults: Guidelines from the Association of the Scientific Medical Societies in Germany. Eur J Anaesthesiol. 2016;33:488-521.

21. Kozek-Langenecker SA, Afshari A, Albaladejo P, Santullano CA, De Robertis E, Filipescu DC, et al. Management of severe perioperative bleeding: guidelines from the European Society of Anaesthesiology. Eur J Anaesthesiol. 2013;30:270-382.

22. Jammer I, Wickboldt N, Sander M, Smith A, Schultz MJ, Pelosi P, et al. Standards for definitions and use of outcome measures for clinical effectiveness research in perioperative medicine: European Perioperative Clinical Outcome [EPCO] definitions: a statement from the ESA-ESICM joint taskforce on perioperative outcome measures. Eur J Anaesthesiol. 2015;32:88-105.

23. Hinz A, Kohlmann T, Stobel-Richter $Y$, Zenger M, Brahler E. The quality of life questionnaire EQ-5D-5L: psychometric properties and normative values for the general German population. Qual Life Res. 2014;23:443-7.

24. Salzwedel C, Puig J, Carstens A, Bein B, Molnar Z, Kiss K, et al. Perioperative goal-directed hemodynamic therapy based on radial arterial pulse pressure variation and continuous cardiac index trending reduces postoperative complications after major abdominal surgery: a multi-center, prospective, randomized study. Crit Care. 2013;17:R191.

25. Ackland GL, Iqbal S, Paredes LG, Toner A, Lyness C, Jenkins N, et al. Individualised oxygen delivery targeted haemodynamic therapy in high-risk surgical patients: a multicentre, randomised, double-blind, controlled, mechanistic trial. Lancet Respir Med. 2015;3:33-41.

26. Pearse RM, Harrison DA, MacDonald N, Gillies MA, Blunt M, Ackland G, et al. Effect of a perioperative, cardiac output-guided hemodynamic therapy algorithm on outcomes following major gastrointestinal surgery: a randomized clinical trial and systematic review. JAMA. 2014;311:2181-90.

\section{Submit your next manuscript to BioMed Central and we will help you at every step:}

- We accept pre-submission inquiries

- Our selector tool helps you to find the most relevant journal

- We provide round the clock customer support

- Convenient online submission

- Thorough peer review

- Inclusion in PubMed and all major indexing services

- Maximum visibility for your research

Submit your manuscript at www.biomedcentral.com/submit 